\title{
Late onset MLD with normal nerve conduction associated with two novel missense mutations in the ASA gene
}

\author{
S Gallo, D Randi, M Bertelli, Al Salviati, M Pandolfo
}

J Neurol Neurosurg Psychiatry 2004;75:655-657. doi: 10.1136/jnnp.2003.017400

Metachromatic leukodystrophy (MLD) rarely has its clinical onset in young adults, with a combination of cognitive and behavioural symptoms and peripheral neuropathy. Here we present an exceptional case with very late onset at 42 years of age and no clinical or neurophysiological sign of peripheral neuropathy. Molecular analysis revealed compound heterozygosity for two novel missense mutations affecting conserved residues in the arylsulphatase $A$ (ASA) sulphatase and carboxyterminal domains, resulting in an $89 \%$ loss of enzymatic activity. This case indicates that MLD needs to be considered in the differential diagnosis of very late onset white matter diseases, even if not accompanied by peripheral nerve involvement.

M etachromatic leukodystrophy (MLD) is an autosomal recessive disease caused by mutations in the arylsulphatase A $(A S A)$ gene. Loss of ASA function leads to sulphatide accumulation and myelin breakdown in the central and peripheral nervous system. The resulting clinical picture is that of a leukodystrophy associated with peripheral neuropathy. ${ }^{1}$

Most MLD cases have infantile or juvenile onset. However, a few patients with adult onset MLD, usually in the second or third decade of life, have been reported. ${ }^{2}$ Psychiatric and behavioural abnormalities are the typical presenting symptoms of adult onset $M L D,{ }^{3}$ followed by progressive dementia, ataxia, spasticity, and seizures. ${ }^{24} \mathrm{~A}$ peripheral demyelinating neuropathy was present in all published cases, with the exception of five patients from three families. ${ }^{5-7}$ A specific ASA missense mutation, Il79S, has been tentatively associated with late onset MLD, particularly when psychiatric symptoms are prominent. ${ }^{8}$ No mutation data are available about the extremely rare patients that presented without peripheral neuropathy.

Here we report a very late onset adult MLD patient with no clinical or neurophysiological evidence of peripheral neuropathy, who is a compound heterozygote for two previously unreported missense mutations in the $A S A$ gene.

\section{METHODS \\ Patient}

A 47 year old male of northern Italian origin, born of nonconsanguineous parents, was evaluated for a progressive dementing illness started at the age of 42 years. The first symptoms consisted of unpredictable and inappropriate behaviour with bursts of aggressiveness. Occasional loss of spatial orientation heralded a progressive cognitive deterioration. Informed consent was obtained for molecular genetic studies.

\section{DNA extraction and PCR amplification}

Genomic DNA was extracted from venous blood using the Puregene kit (Gentra) according to the manufacturer's instructions. Primers used for amplifying genomic ASA sequences were designed according to the genomic sequence of the ASA gene (GenBank accession no. X52150). ${ }^{9}$ All reactions were performed in $50 \mu \mathrm{l}$ volumes. The PCR mixture contained $10 \mathrm{mmol} / \mathrm{l}$ Tris- $\mathrm{HCl} \mathrm{pH} \mathrm{8.3,} 50 \mathrm{mmol} / \mathrm{l} \mathrm{KCl}$, $1.5 \mathrm{mmol} / \mathrm{l} \mathrm{MgCl}_{2}, 0.2 \mathrm{mmol} / \mathrm{l}$ each dNTP, $0.6 \mu \mathrm{mol} / \mathrm{l}$ each forward and reverse primer, $1.25 \mathrm{U}$ of AmpliTaq Gold (Applied Biosystems, Foster City, CA, USA), and 200 ng of genomic DNA template. Thermal cycling was carried out with an initial denaturation of $94^{\circ} \mathrm{C}$ for 5 minutes then 30 cycles of denaturation at $94^{\circ} \mathrm{C}$ for 30 seconds, annealing at $60^{\circ} \mathrm{C}$ for 30 seconds, extension at $72^{\circ} \mathrm{C}$ for 45 seconds, and a final extension at $72^{\circ} \mathrm{C}$ for 10 minutes.

\section{DNA sequencing and analysis}

Amplified fragments were purified of excess primers and nucleotides using a GFX ${ }^{\mathrm{TM}}$ PCR DNA and Gel Band Purification kit (Amersham Biosciences, Amersham, Buckinghamshire, UK) and resuspended in $20 \mu \mathrm{l}$ of distilled water according to the manufacturer's instructions. Purified PCR products were sequences from the antisense and sense strands obtained using the same primers as those used in the PCR amplification step and the DYEnamic ${ }^{\mathrm{TM}}$ ET Terminator Cycle Sequencing kit (Amersham Biosciences). Cycle sequencing was performed according to the manufacturer's instructions, after which sequencing products were purified on AutoSeq ${ }^{\mathrm{TM}}$ G-50 columns (Amersham Biosciences). The sequencing reagents were analysed on an automatic ABI Prism 377 DNA Sequencer (Perkin-Elmer Applied Biosystems).

\section{PCR amplification of specific alleles}

To confirm the two mutations found we used allele specific oligonucleotides that differed only in the $3^{\prime}$ terminal base. For the $878 \mathrm{G} \rightarrow \mathrm{A}$ mutation we used the primers ARSA 878G (ACCATGCGTATGTCCCGAGGCGG), corresponding to the wild type allele, and ARSA 878A (ACCATGCGTATGTCCCGAGGCGA) corresponding to the mutant allele. Amplification was carried out using the antisense primer ARSA 5/6R. For the $1465 \mathrm{~T} \rightarrow \mathrm{G}$ mutation we used the primers ARSA 1465T (GGGTGCAGC GACGATGACA) (wild type) and ARSA 1465G (GGGTG CAGCGACGATGACC) (mutant). Amplification was carried out using the primer ARSA 8F. Both PCR were performed under the same conditions as mentioned above.

Abbreviations: MLD, metachromatic leukodystrophy; MRI, magnetic resonance imaging 


\section{RESULTS}

\section{Case report}

The patient was hospitalised at 46 years of age for diagnostic investigation. Brain magnetic resonance imaging (MRI) showed a diffusely increased T2 signal in the white matter, most prominent in the frontal lobes and near the ventricles, and a thin corpus callosum (fig 1). Electromyography and nerve conduction studies did not reveal any abnormality (table 1). Multimodal evoked potentials (visual, auditory, and somatosensory) also were within normal limits. The electroencephalogram showed widespread epileptiform activity. Based on the above clinical findings, a screening for adult onset leukodystrophies was carried out. Plasma aminoacids, urinary organic acids, very long chain fatty acids, and betagalactocerebrosidase were all within normal limits. ASA activity in leukocytes was $11.3 \%$ of the mean of the controls. A positive essay for urinary sulphatides confirmed the diagnosis of MLD.

At the age of 47 years, the patient was examined by one of the authors (MP). He was quiet and collaborating, but disoriented in time and space. A severe concentration and attention deficit, lack of motor persistence, and lack of perseveration were evident. In addition to these signs of frontal lobe involvement, a moderate memory deficit, acalculia, anosognosia, visuospatial deficits, and a mild comprehension deficit associated with poor verbal output indicated a more global brain dysfunction. There was no
Table 1 Results of nerve conduction studies

\begin{tabular}{|c|c|c|c|c|}
\hline & Lat (ms) & Amp (mV) & $\mathrm{CV}(\mathrm{m} / \mathrm{s})$ & $\mathrm{F}-\mathrm{M}(\mathrm{ms})$ \\
\hline \multicolumn{5}{|l|}{ Motor nerves } \\
\hline Left ulnar & & & & 24.1 \\
\hline Wrist-abductor V & 2.3 & 14.5 & & \\
\hline Elbow-wrist & 6.2 & 14.6 & 59.0 & \\
\hline Right tibial & & & & 42.5 \\
\hline Ankle-abductor allucis & 3.2 & 18.8 & & \\
\hline Popliteal fossa-ankle & 12.2 & 16.7 & 47.8 & \\
\hline Right peroneal & & & & 40.8 \\
\hline Ankle-pedidius & 4.0 & 17.2 & & \\
\hline Fibular head-ankle & 10.3 & 17.5 & 46.8 & \\
\hline \multicolumn{5}{|l|}{ Sensory nerves } \\
\hline Left median & 2.5 & 59 & 58.0 & \\
\hline Right sural & 3.0 & 20 & 46.7 & \\
\hline Left sural & 3.1 & 15 & 45.2 & \\
\hline
\end{tabular}

ataxia and no focal motor or sensory deficit. Tendon reflexes were symmetrically increased in the four limbs and a bilateral Babinski sign was present.

\section{Molecular genetic studies}

The patient was found to be a compound heterozygote for two novel missense mutations in ASA, G293D in exon 5, and C489G mutation in exon 8, and heterozygous for the N350S pseudodeficiency polymorphism in exon 6. The G293D
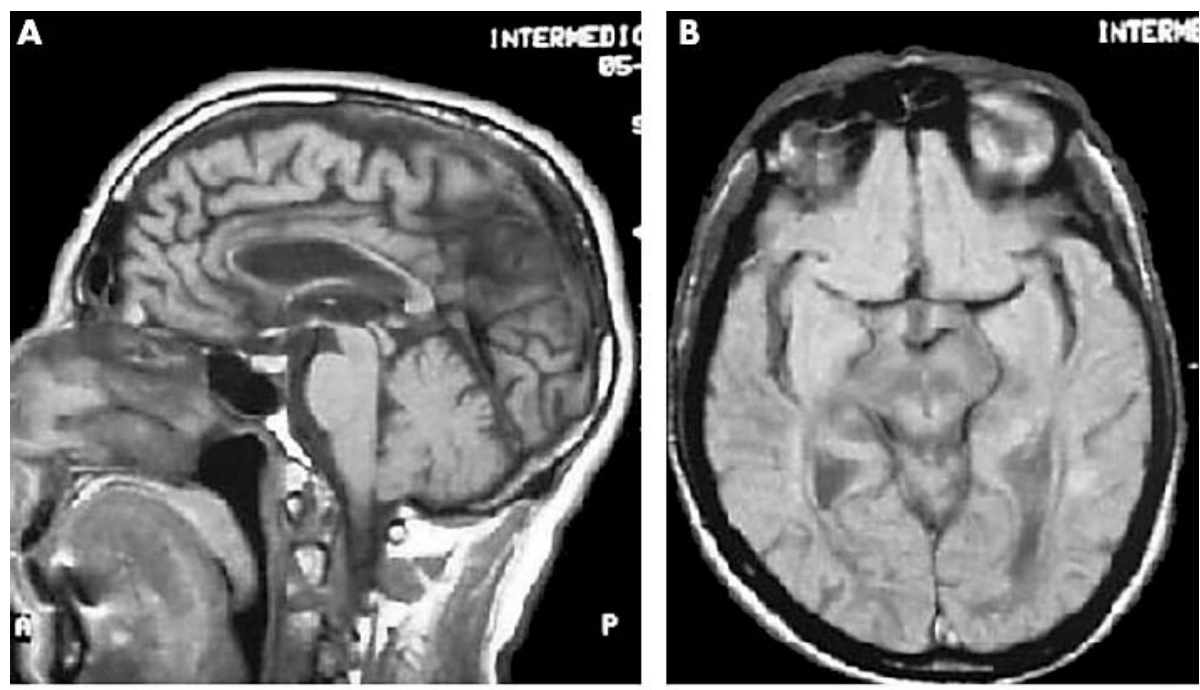

Figure $1 \quad \mathrm{Tl}$ weighted (a) and FLAIR (B-D) brain MRI images showing a thin corpus callosum (A) and signal hyperintensity of the periventricular (C) and frontal (D) white matter with relative sparing of the posterior white matter (B).
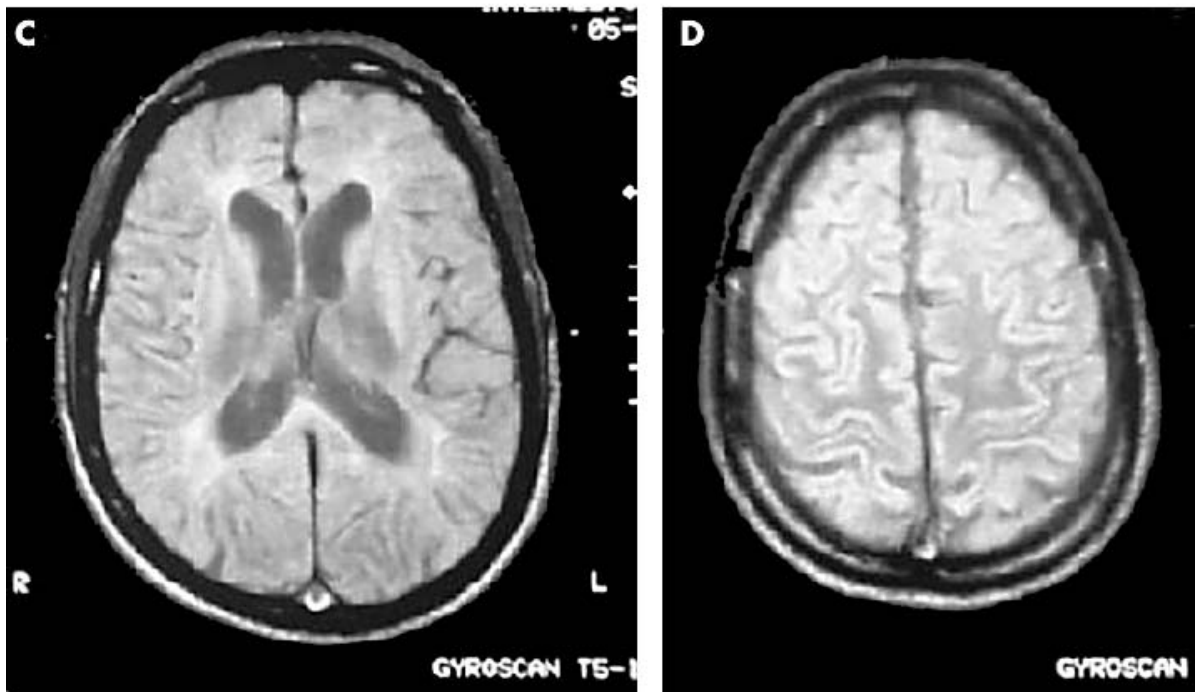
mutation is localised to the ASA sulphatase domain, in a position that is conserved in other sulphatases and in alkaline phosphatase. The C489G mutation involves a highly conserved cysteine residue near the carboxyl terminus. Analysis of the patient's mother and maternal uncle revealed both the G293D mutation and the N350S polymorphism, showing that they occurred in a cis configuration. Using PCR amplification of specific alleles, the paternal uncle was not found to carry the C489G mutation. Neither mutation was found in more than 100 unrelated healthy controls from the same region of northern Italy.

\section{DISCUSSION}

The clinical presentation of our patient was remarkable because of the absence of detectable peripheral nervous system involvement and because of the exceptionally late onset of symptoms in the fifth decade of life. Onset with psychiatric symptoms was otherwise typical for adult onset MLD. ${ }^{3}$ Behavioural and cognitive abnormalities still dominated the clinical picture 5 years after onset. At this stage the patient was clearly demented, with global neuropsychological deficits, although the most severe deficits involved frontal lobe functions. Motor deficits and ataxia were markedly absent. The clinical picture was in agreement with the MRI finding of a diffuse leukoencephalopathy predominantly affecting the frontal lobes and the periventricular white matter, but not sparing any part of the brain.

The absence of peripheral nervous system involvement is very unusual in adult onset MLD. Two among the 14 cases described by Kappler et $a l^{10}$ had no detectable peripheral nerve involvement in the very early symptomatic stage of the disease, but only the patients reported by Seidel et al (one case), ${ }^{5}$ Brion et al (one case), ${ }^{6}$ and Cengiz et al (three cases in one family) ${ }^{7}$ continued to have no detectable neuropathy several years into the clinical course.

To our knowledge, our patient is the first case of adult MLD without neuropathy for whom the causative mutations in the ASA gene have been identified. The presence of the pseudodeficiency polymorphism confounded the interpretation of the biochemical analysis of our patient and his family, a situation that has been previously reported. ${ }^{11}$ We could in any case demonstrate that our patient has the biochemical abnormality of MLD by showing an increased urinary excretion of sulphatides.

Microdeletions and point mutations in ASA may cause MLD. Milder cases usually have missense mutations that allow some residual enzyme activity. The pathological nature of the two novel missense mutations we identified in our patient was supported by the non-conservative amino acid changes, by the involvement of conserved residues in the ASA protein and by their absence in more than 100 controls from the same population. However, a straightforward genotype-phenotype correlation remains difficult. Despite the confounding effect of the associated pseudodeficiency, it is clear that the patient's missense mutations led to a less severe enzyme defect than in typical infantile MLD. ${ }^{10}$ Accordingly, urinary excretion of sulphatides is also less important, as evaluated by a semi-quantitative essay. However, mild disease can only be part of the explanation for this peculiar phenotype. Intriguingly, the opposite phenotype of exclusive peripheral nerve involvement has also been reported in cases of mild late onset MLD. ${ }^{12}{ }^{13}$ The causative mutation, a homozygous aminoacid change (T286P), was determined for one of these patients. ${ }^{13}$

Our observation further extends the phenotype of adult onset MLD. This condition should be tested in all adult patients presenting with an otherwise unexplained leukoencephalopathy, particularly if the frontal lobes are most affected, even when onset is as late as the fifth decade and there is no detectable peripheral nerve involvement.

\section{ACKNOWLEDGEMENTS}

This work was supported by the Fondazione BNC, Rome, Italy.

\section{Authors' affiliations}

S Gallo, D Randi, M Bertelli, Istituto BIRD Europe, Vicenza, Italy A Salviati, Department of Neurology, University of Verona, Italy M Pandolfo, Department of Neurology, Hôpital Erasme, Université Libre de Bruxelles, Brussels, Belgium

Competing interests: none declared

Correspondence to: $\operatorname{Dr} M$ Pandolfo, Service de Neurologie, Hôpital Erasme, Route de Lennik 808, 1070 Bruxelles, Belgium; massimo.pandolfo@ulb.ac.be

Received 24 April 2003

In revised form 25 July 2003

Accepted 6 August 2003

\section{REFERENCES}

1 Kolodny EH. Metachromatic leukodystrophy and multiple sulfatase deficiency: sulfatide lipidosis. In: Scriver CR, Beaudet AL, Sly WS, Valle D, eds. The metabolic basis of inherited disease, 6th edn. New York: McGraw-Hill, 1995:2693-739.

2 Hageman AT, Gabreels FJ, De Jong JG, et al. Clinical symptoms of adult metachromatic leukodystrophy and arylsulfatase A pseudodeficiency. Arch Neurol 1995;52:408-13.

3 Hyde TM, Ziegler JC, Weinberger DR. Psychiatric disturbances in metachromatic leukodystrophy. Arch Neurol 1992;49:401-6.

4 Bauman N, Masson M, Carreau V, et al. Adult forms of metachromatic leukodystrophy: clinical and biochemical approach. Dev Neurosci 1991; 13:211-15.

5 Seidel D, Goebel HH, Scholz W. Late onset metachromatic leukodystrophy: diagnostic problems elucidated by a case report. J Neurol 1981;226:1 19-24.

6 Brion S, Mikol J, Graveleau J. Leucodystrophie métachromatique de l'adulte jeune: étude clinique, biologique et ultrastructurale. Rev Neurol 1970;122:161-76.

7 Cengiz N, Özbenli T, Onar M, et al. Adult metachromatic leukodystrophy: three cases with normal conduction velocities in a family. Acta Neurol Scand 2002; 105:454-7.

8 Tylki-Szymanska A, Berger J, Loschl B, et al. Late juvenile metachromatic leukodystrophy $(M L D)$ in three patients with a similar clinical course and identical mutation on one allele. Clin Genet 1996;50:287-92.

9 Kreysing J, von Figura K, Gieselmann V. Structure of the arylsulfatase A gene. Eur J Biochem 1990;191:627-31.

10 Kappler J, Leinekugel P, Conzelmann E, et al. Genotype-phenotype relationship in various degrees of arylsulfatase A deficiency. Hum Genet $1991 ; 86: 463-70$.

11 Francis GS, Bonni A, Shen N, et al. Metachromatic leukodystrophy: Multiple non-functional and pseudodeficiency alleles in a pedigree: problems with diagnosis and counseling. Ann Neurol 1993;34:212-18.

12 Fressinaud C, Vallat JM, Masson M, et al. Adult onset metachromatic leukodystrophy presenting as isolated peripheral neuropathy. Neurology 1992:42:1396-8.

13 Felice KJ, Lira GM, Natowicz M. Adult onset metachromatic leukodystrophy: a gene mutation with isolated polyneuropathy. Neurology 2000;55:1036-9. 